\title{
An Arbitrary Lagrangian Eulerian Solver for Shock Imploding Fissile Materials
}

\author{
Eric Cervi, Antonio Cammi \\ Politecnico di Milano, Via La Masa 34, 20156 Milan, Italy \\ eric.cervi@polimi.it, antonio.cammi@polimi.it \\ https://dx.doi.org/10.13182/T31112
}

\section{INTRODUCTION}

A multiphysics finite volume method (FVM) solver, coupling neutronics and shock physics, is under development at Politecnico di Milano for the analysis of shock imploding fissile materials [1].

This research activity aims at providing a computational tool to perform preliminary safety assessment of subcritical experiments [2] and, more in general, to carry out criticality safety evaluations in case of strongly energetic events (such as chemical explosions) involving fissile material [3].

To this aim, a multi-group $\mathrm{SP}_{3}$ neutron transport model is implemented for neutronics, suitable to provide an accurate description of reactivity and of the neutron flux in small and strongly leakage-dominated systems. On the other hand, the shock physics model implements mass, momentum and energy balance equations and the Mie-Gruneisen equation of state, in order to describe the propagation of strong shockwaves in solid materials. The shock physics module also implements a dynamic mesh, in order to catch the solid material deformation induced by shock compression. In addition, the balance equations are written in an Arbitrary Lagrangian-Eulerian (ALE) form, in order to preserve the computational mesh quality in case of strong deformations.

Different shock physics codes are available in literature, (see, e.g., [4,5]) but none of them implements a neutron transport module. Codes are also available to study non-linear wave propagations in liquid fuel reactors [6], but they are not suitable for shock compression of solids. In this regard, the aim of the present activity is to fill the gap, coupling neutronics and shock physics model in the same simulation environment. In more details, the purpose of this report is to present an ALE algorithm specifically developed for coupled neutronics and shock physics problems. The proposed algorithm is tested on two different case studies - a subcritical and a supercritical imploding cylinder - highlighting its advantages with respect to a purely Lagrangian approach.

\section{Modelling approach}

In this section, the structure of the solver and the multiphysics coupling strategy are described. At each time step, the systems neutronics and shock physics are solved in two different iterative cycles, as shown in Figure 1. The temperature and density calculated by the shock physics module are passed to the neutronics one in order to evaluate cross sections. In turn, the fission power calculated by the neutronics solver appears as a source term in the energy equation in the shock physics module. External iterations between the neutronics and shock physics cycles are performed, to solve the non-linearities between the two physics. The neutronics and the shock physics models are briefly described in the following subsections. For more details, the reader is referred to [1].

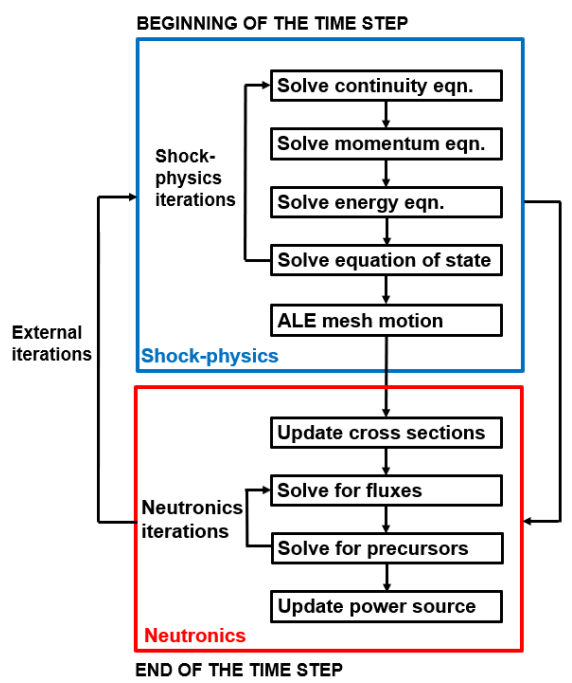

Fig. 1. Solver structure and coupling strategy.

The shock physics model

The implemented shock physics model is based on the so called "hydrodynamic approximation". For very high pressures (above 5-10 GPa), the shear stresses become negligible and the solid response to shock compression is similar to that of an inviscid, compressible fluid [7]. Thanks to this approximation, the stress tensor and thermal conduction can be neglected in the conservation equations, which read as follows:

$$
\begin{gathered}
\frac{\partial \rho}{\partial t}+\nabla \cdot[\rho(\boldsymbol{u}-\boldsymbol{w})]=0 \\
\frac{\partial(\rho \boldsymbol{u})}{\partial t}+\nabla \cdot[\rho \boldsymbol{u}(\boldsymbol{u}-\boldsymbol{w})]=-\nabla p+\boldsymbol{b} \\
\frac{\partial(\rho h)}{\partial t}+\nabla \cdot[\rho h(\boldsymbol{u}-\boldsymbol{w})]=\frac{D p}{D t}+\dot{q}
\end{gathered}
$$

The balance equations are written in an Arbitrary Lagrangian-Eulerian (ALE) form: the mesh vertices can be moved with an arbitrary velocity $\boldsymbol{w}$, to preserve the mesh quality in case of strong distortions. This velocity is included 
in the advective terms of the equations in order to preserve the balances.

Under the hydrodynamic approximation, the material behavior can be described by the Mie-Gruneisen equation of state [7]:

$$
p-p_{H}=\frac{\gamma(v)}{v}\left(e-e_{H}\right)
$$

where $\gamma$ is the Gruneisen parameter, while $p_{H}$ and $e_{H}$ are the pressure and internal energy lying on a Hugoniot curve [7], which depends on the specific material and must be known experimentally. Validation of the proposed shock physics model against experimental data can be found in [1].

\section{The neutronics model}

A multi-group SP3 transport model is implemented for neutronics. Compared to diffusion approaches, a transport model is more suitable for the description of small systems, in which neutron leakages have a strong impact on reactivity. In this regard, the SP3 equations are selected as a trade-off between model accuracy and computational cost:

$$
\begin{gathered}
\frac{1}{v_{i}} \frac{\partial \Phi_{0, i}}{\partial t}=\nabla \cdot D_{0, i} \nabla \Phi_{0, i}-\Sigma_{r, i}\left(\Phi_{0, i}-2 \varphi_{2, i}\right)+S_{n, i}(1-\beta) \chi_{p, i} \\
+S_{d} \chi_{d, i}+S_{s, i}+\frac{2}{v_{i}} \frac{\partial \varphi_{2, i}}{\partial t} \\
\frac{9}{5} \frac{1}{v_{i}} \frac{\partial \varphi_{2, i}}{\partial t}=\nabla \cdot D_{2, i} \nabla \varphi_{2, i}-\Sigma_{t 2, i} \varphi_{2, i}+\frac{2}{5} \Sigma_{r, i}\left(\Phi_{0, i}-2 \varphi_{2, i}\right) \\
-\frac{2}{5} S_{n, i}(1-\beta) \chi_{p, i}-\frac{2}{5} S_{d} \chi_{d, i}-\frac{2}{5} S_{s, i}+\frac{2}{5} \frac{1}{v_{i}} \frac{\partial \Phi_{0, i}}{\partial t}
\end{gathered}
$$

Where $S_{n}, S_{s}$ and $S_{d}$ are the fission neutron, scattering neutron and delayed neutron source terms [1], respectively, while:

$$
\Phi_{0, i}=\varphi_{0, i}+2 \varphi_{2, i}
$$

with $\varphi_{0, i}$ and $\varphi_{2, i}$ being the zeroth and the second moment neutron fluxes.

Balance equations for precursor densities are included into the neutronics model as follows:

$$
\frac{\partial c_{k}}{\partial t}+\nabla \cdot\left[c_{k}(\boldsymbol{u}-\boldsymbol{w})\right]=\beta_{k} \sum_{i} \bar{v} \Sigma_{f, i} \varphi_{i}-\lambda_{k} c_{k}
$$

In addition, a power iteration routine, based on the $k$ eigenvalue method, is also implemented for the estimation of the multiplication factor. The present $\mathrm{SP}_{3}$ model has been tested and verified in [8,9], while its limits for the present application will be discussed in parallel publications.

\section{An ALE algorithm for coupled neutronics and shock physics problems}

In the Arbitrary Lagrangian-Eulerian (ALE) formulation [10], an arbitrary mesh velocity $\boldsymbol{w}$ appears in the avvective terms of the balance equations. This velocity can be determined with various algorithms, in order to preserve the mesh quality in case of strong mesh distortions. If $\boldsymbol{w}=\mathbf{u}$, the mesh follows the motion of the solid particles, reducing to a purely Lagrangian approach, while if $\boldsymbol{w}=0$ the mesh is fixed, reducing to a purely Eulerian approach. In general, Lagrangian approaches are more accurate, especially when the motion of sharp surfaces or material interfaces has to be described, but they can suffer from mesh distortion and tangling issues that can make the problem incomputable. On the other hand, Eulerian approaches are less accurate, due to numerical diffusion and dispersion, but are not affected by distortion issues since the mesh does not move.

To overcome the issues of purely Lagrangian and Eulerian descriptions, ALE techniques have been developed to combine the best feature of both the approaches. Thanks to the freedom in moving the mesh offered by the ALE approach, large distortions can be handled, while preserving the accurate description of interfaces typical of a purely Lagrangian approach.

Many ALE algorithms are available in literature to determine the arbitrary mesh velocity $\boldsymbol{w}$. In this work, a new ALE algorithm, specifically developed for the analysis of shock imploding fissile bodies is developed. In more details, the algorithm is designed to possess the following characteristics, as summarized by Fig. 2:

1. At the domain center, where the imploding shockwave converges, the high values reached by pressure can lead to mesh tangling. Therefore, a Eulerian approach is desired in the central region of the imploding body.

2. At the boundary, high precision is needed to describe the motion of the system surface, in order to correctly estimate neutron leakages and reactivity in turn. At the same time, pressure is lower than in the center of the domain, and a Lagrangian approach can be afforded.

3. The neutron flux is lower at the boundary, where a Lagrangian approach is preferable, while it's higher in the center, where a Eulerian approach is required. Therefore, the algorithm should be able to rely on the neutron flux as a criterion to switch between the Eulerian and Lagrangian point of views.

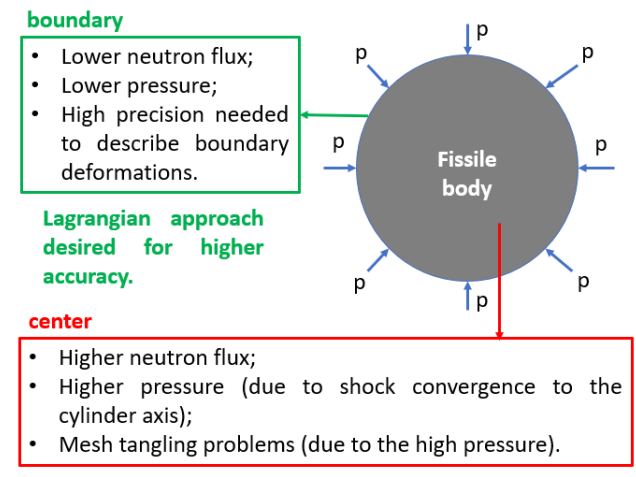

Lagrangian approach unfeasible due to mesh tangling. Eulerian approach desired to preserve the mesh quality.

Fig. 2. Desired features of the proposed ALE algorithm. 
In the light of these requirements, the following ALE algorithm is developed. Firstly, a function $f_{A L E}$ is introduced, which is linked to the neutron flux by obeying to the following diffusion-like equation:

$$
\nabla \cdot D \nabla f_{A L E}-\Sigma_{a} f_{A L E}+\bar{v} \Sigma_{f} \varphi_{0, \text { total }}=0
$$

with $f_{A L E}=0$ at the boundary. Note that the total flux appears in the fission term of Eq. (9), otherwise the solution would be $f_{A L E} \equiv 0$. Then, $f_{A L E}$ is normalized with respect to its maximum value:

$$
F_{A L E}=\frac{f_{A L E}}{\max \left(f_{A L E}\right)}
$$

Due to its definition and to the zero boundary conditions imposed to $f_{A L E}$, the normalized function $F_{A L E}$ is bounded between 0 and $1 . F_{A L E}$, which is linked to the neutron flux by Eqs. (9) and (10), is chosen as criterion to switch between the Lagrangian and the Eulerian point of views. To this aim, the arbitrary velocity $\boldsymbol{w}$ is defined as follows:

$$
\boldsymbol{w}=\boldsymbol{u}\left(1-F_{A L E}\right)
$$

According to Eq. (11), $\boldsymbol{w}=\boldsymbol{u}$ (Lagrangian approach) at the boundary, where $F_{A L E}=0$, while $\boldsymbol{w}=0$ (Eulerian approach) in the center of the domain, where $F_{A L E}=1$.

In general, a mixed Lagrangian and Eulerian approach is enough to avoid mesh tangling in the central region of the domain, without requiring a (less accurate) purely Eulerian point of view. Therefore, Eq. (11) is slightly modified by introducing an under-relaxation coefficient $r_{A L E}=0 \sim 1$ :

$$
\boldsymbol{w}=\boldsymbol{u}\left(1-r_{A L E} F_{A L E}\right)
$$

After several testing, it has been observed that with $r_{A L E}=0.2 \sim 0.5$ (depending on the specific material and geometry) mesh tangling issues are avoided and results are only negligibly affected, compared to the more accurate Lagrangian approach. In particular, the validation of the shock physics model carried out in [1] still holds using the present ALE algorithm, for many different materials.

\section{RESULTS}

The ALE approach described in the previous section is now tested on two case studies:

1. A 2D uranium infinite cylinder, enriched at $95 \%$ in ${ }^{235} \mathrm{U}$, with $3 \mathrm{~cm}$ radius and initial multiplication factor $k_{\text {eff }}=0.56650$;

2. A 2D uranium infinite cylinder with same enrichment, $6 \mathrm{~cm}$ radius and initial multiplication factor $k_{\text {eff }}=0.97911$.

A pressure of $30 \mathrm{GPa}$ is applied to the lateral surface of the two cylinders. Using a purely Lagrangian approach, the simulation of both the implosions blows up just before the shock converges at the cylinder axis, due to mesh tangling (see Figs. 3 and 4).

The evolution of the pressure profile for the $3 \mathrm{~cm}$ cylinder is presented in Fig. 3. At $t=8.40 \mu \mathrm{s}$, as the shockwave converges to the cylinder axis, pressure abruptly increases up to about $18 \mathrm{Mbar}$, and maximum compression is achieved. The multiplication factor grows to $k_{e f f}=$ 0.62407, still far below criticality, due to the small dimensions of the cylinder. Afterwards, the shockwave returns towards the cylinder surface, pressure decreases, and the cylinder expands.

On the other hand, using a purely Lagrangian approach, the problem becomes incomputable around $t=8.00 \mu \mathrm{s}$, when the maximum pressure is only 1.2 Mbar.
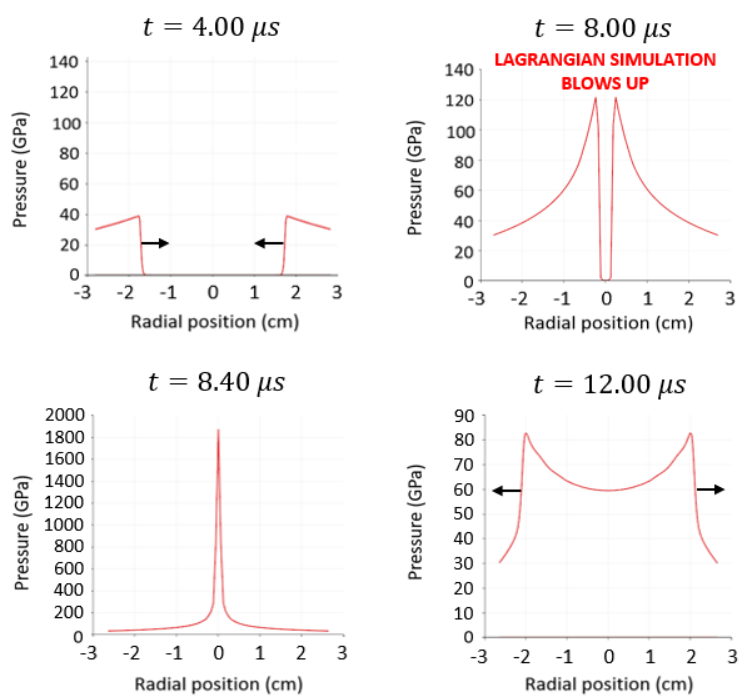

Fig. 3. ALE simulation of the $3 \mathrm{~cm}$ cylinder implosion.

A completely different situation is observed for the $6 \mathrm{~cm}$ cylinder (Fig. 4). At maximum compression, achieved at $t=$ $16.80 \mu \mathrm{s}$, the system is strongly supercritical, with multiplication factor $k_{e f f}=1.11105$. A bell-shaped pressure profile, due to the fission power heating, is superimposed to the pressure peak due to shock convergence, leading to a pressure of $65 \mathrm{Mbar}$ at the cylinder axis. Due to the sudden heating, the cylinder rapidly expands, reaching its original dimensions at $t=16.95 \mu \mathrm{s}$. Despite the expansion, pressure continues to increase since fission reactions are still releasing energy into the system. In particular, at $t=$ $16.95 \mu \mathrm{s}$ pressure reaches a peak value of $103 \mathrm{Mbar}$.

In the light of these results, the proposed ALE algorithm proves to be effective in avoiding mesh tangling issues, allowing to simulate the compression and the subsequent expansion of both subcritical and supercritical systems. A significant improvement is achieved with respect to a purely Lagrangian approach, which is affected by mesh tangling issues even before the shockwave converges to the cylinder axis. 


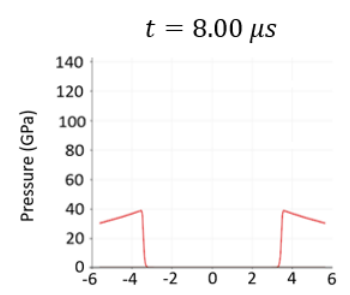

Radial position $(\mathrm{cm})$

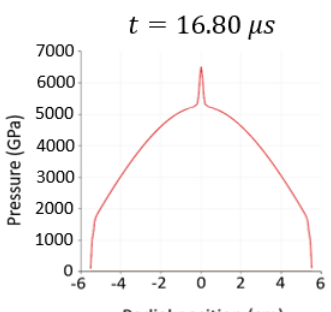

Radial position $(\mathrm{cm})$
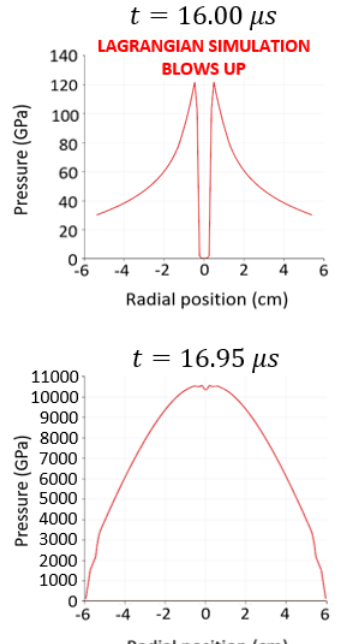

Radial position $(\mathrm{cm})$

Fig. 4. ALE simulation of the $6 \mathrm{~cm}$ cylinder implosion.

\section{Conclusions}

In this work, a coupled neutronics and shock physics solver is extended, implementing an Arbitrary Lagrangian Eulerian (ALE) algorithm specifically developed for the analysis of shock imploding fissile bodies. The proposed method shows significant improvements compared to purely Lagrangian approaches, allowing to simulate much stronger compressions without incurring in mesh tangling issues that would make the problem incomputable.

Thanks to its capability to simulate both critical and subcritical systems, the present solver constitutes a promising tool for the analysis of coupled neutronics and shock physics problems. In more details, this solver can be a useful tool to support the design of subcritical experiments and to assess their safety and, more in general, to study reactivity accidents initiated by strongly energetic events such as chemical explosions. The improvement of the neutronics model, by means of the implementation of a discrete ordinate subsolver, will be discussed in parallel publications.

\section{NOMENCLATURE}

\section{Latin symbols}

$\begin{array}{ll}\boldsymbol{b} & \text { Body force, } \mathrm{kg} \mathrm{m}^{-2} \mathrm{~s}^{-2} \\ c & \text { Precursor density, } \mathrm{m}^{-3} \\ F_{A L E} & \text { Normalized ALE function, - } \\ f_{A L E} & \text { ALE function, } \mathrm{m}^{-2} \mathrm{~s}^{-1} \\ h & \text { Enthalpy, } \mathrm{J} \mathrm{kg}^{-1} \\ p & \text { Pressure, } \mathrm{Pa} \\ \dot{q} & \text { Power source, } \mathrm{J} \mathrm{s}^{-1} \mathrm{~m}^{-1} \\ r_{A L E} & \text { ALE under-relaxation factor, - } \\ t & \text { Time, } \mathrm{s} \\ \boldsymbol{u} & \text { Material velocity, } \mathrm{m} \mathrm{s}^{-1} \\ v & \text { Specific volume, } \mathrm{m}^{3} \mathrm{~kg}^{-1} \\ v_{i} & \text { Neutron velocity, } \mathrm{m} \mathrm{s}^{-1} \\ \boldsymbol{w} & \text { Arbitrary mesh velocity, } \mathrm{m} \mathrm{s}^{-1} \\ \text { Greek symbols } \\ \beta & \text { Delayed neutron fraction, - }\end{array}$

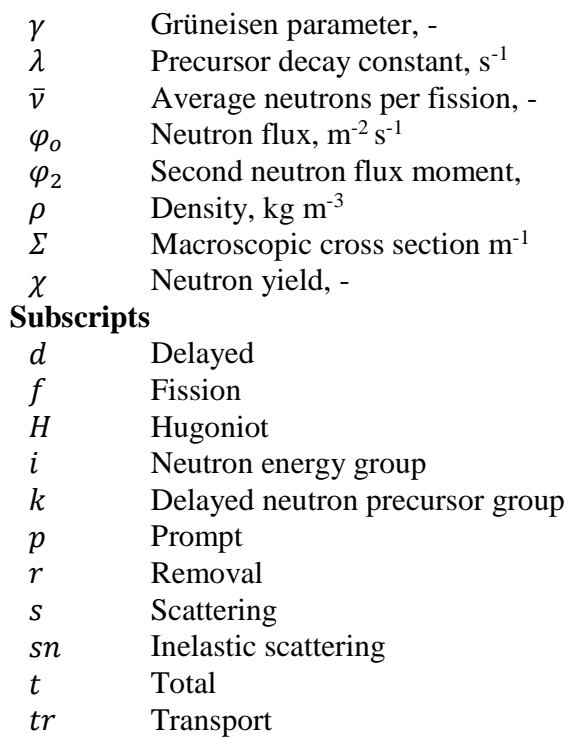

\section{REFERENCES}

1. Cervi, E., Lorenzi, S., Luzzi, L., Cammi, A., "An OpenFOAM solver for Criticality Safety Assessment in Dynamic Compression Events", Transactions of the American Nuclear Society, 119, p. 855-858 (2018).

2. Conrad, D.C., Science \& Technology Review 2000 (2000). 3. "DOE Technical Standard, Preparing critical safety evaluations at department of energy nonreactor facilities" (2017).

4. McGlaun, J.M., Thompson, S.L., "CTH: A threedimensional shock wave physics code", International Journal of Impact Engineering, 10, 351-360 (1990).

5. Summers, R.M., Peery, J.S., Wong, M.W., Herten Jr., E.S., Trucano, T.G., Chhabildas, L.C., "Recent progress in ALEGRA development and application to ballistic impacts", International Journal of Impact Engineering, 20, p. 779-788 (1997).

6. Cervi, E., Lorenzi, S., Cammi, A., Luzzi, L., "Development of a multiphysics model for the study of fuel compressibility effects in the Molten Salt Fast Reactor", Chemical Engineering Science, 193, p. 379-393 (2019).

7. Davison, L., Fundamentals of Shock Wave Propagation in Solids, Springer-Verlag Berlin Heidelberg (2008).

8. Cervi, E., Lorenzi, S., Cammi, A., Luzzi, L., "Development of an SP3 neutron transport solver for the analysis of the Molten Salt Fast Reactor", Nuclear Engineering and Design, 346, p. 209-219 (2019).

9. Cervi, E., Lorenzi, S., Luzzi, L., Cammi, A., "Multiphysics analysis of the MSFR helium bubbling system: A comparison between neutron diffusion, SP3 neutron transport and Monte Carlo approaches", Annals of Nuclear Energy, 132, p. 227-235 (2019).

10. Hirt, C.W., Amsden, A.A., Cook, J.L., "An arbitrary Lagrangian-Eulerian computing method for all flow speeds", Journal of Computational Physics, 14, p. 227-253 (1974). 